\title{
GESTIÓN DE POLÍTICAS DE INVENTARIO EN EL ALMACENAMIENTO DE MATERIALES DE ACERO PARA LA CONSTRUCCIÓN
}

\section{INVENTORY POLICY MANAGEMENT IN THE STORAGE OF STEEL MATERIALS FOR CONSTRUCTION}

\author{
Arturo Contreras Juárez ${ }^{1,}$, Catya Atziry Zuñiga ${ }^{2}$, José Luis Martínez Flores ${ }^{3}$, \\ Diana Sánchez Partida ${ }^{4}$
}

\section{RESUMEN}

El control de inventarios es de vital importancia, por el monto de la inversión que representa y por el grado de dificultad que implica una administración financiera efectiva, para no incurrir en altos costos de almacenamiento. Este artículo presenta el uso del modelo de lote económico de pedido (Economic Order Quantity -EOQ) y el modelo de revisión continua con demanda incierta y con desviación estándar (q, R) para establecer políticas de inventarios en la demanda de materiales de acero para la construcción, comercializados en una empresa de aceros tomando en cuenta la variabilidad de la demanda y los tiempos de suministro de la aplicación de los modelos, quedan establecidas las cantidades optimas a ordenar de los materiales con respecto al tiempo de duración del ciclo de pedido, generando un ahorro del $30 \%$ en los costos logísticos totales e incrementando el nivel de servicio de un $69 \%$ a un $90,4 \%$. El empleo de los modelos propuestos brinda el soporte a las decisiones que han de tomarse en los niveles de inventario de los materiales, minimizando los costos logísticos totales y mejorando la calidad de servicio al cliente.

Palabras Clave: Acero, Política de inventario, Determinístico, Probabilístico, Modelo de lote económico de pedido.

\section{ABSTRACT}

The control of inventories is of vital importance, for the amount of investment it represents and for the degree of difficulty involved in effective financial management, so as not to incur

\footnotetext{
${ }^{1}$ Profesor-Investigador, Departamento de Ingeniería en Logística y Transportes, Universidad Politécnica de Tlaxcala Región Poniente, Tlaxcala México. arturocontreras@uptlaxponiente.edu.mx. orcid.org/0000-0002-7192-3596

2Profesora-investigadora, Departamento de Posgrados, Universidad Aeronáutica en Querétaro, Querétaro, México. catya.zuniga@unaq.edu.mx. orcid.org/0000-0003-3901-9078

${ }^{3}$ Investigador. Puebla, México. joseluis.martinez01@upaep.mx. orcid.org/0000-0003-2986-469X

${ }^{4}$ Profesora-investigadora, Posgrado en Logística y Dirección de la Cadena de Suministro, Universidad Popular Autónoma del Estado de Puebla. Puebla, México. diana.sanchez@upaep.mx. orcid.org/0000-0001-5771-1362
} 
high storage costs. This article presents the use of the economic order lot model (Economic Order Quantity -EOQ) and the continuous review model with uncertain demand and with standard deviation ( $q, R$ ) to establish inventory policies in the demand for steel materials for construction marketed in a steel company taking into account the variability of demand and supply times. From the application of the models, the optimal quantities to the order of the materials are established with respect to the duration of the order cycle, generating a $30 \%$ savings in total logistics costs and increasing the level of service from $69 \%$ to $90,4 \%$. The use of the proposed models provides support to the decisions that have to be taken in the inventory levels of the materials minimizing the total logistics costs and improving the quality of customer service.

Keywords: Steel, Politics of inventory, Deterministic, Probabilistic, Model of economic lot of order.

\section{INTRODUCCIÓN}

La industria del acero es una de las más importantes a nivel mundial por ser proveedora de materia prima para una infinidad de industrias, tales como el ramo automotriz, construcción, alimentos, electrodomésticos, maquinaria pesada entre otras, tan sólo en 2014 , el $51 \%$ de su producción mundial se empleó en la construcción de viviendas e infraestructura (Vásquez \& Abarca, 2018).

Entre sus ventajas del acero para la construcción se encuentra la resistencia, limpieza, posibilidad de reciclaje y facilidad de armado. A medida que pasa el tiempo, se han requerido de menores tiempos de construcción, mayores facilidades de montaje, mejores materiales a precios razonables para generar un impacto financiero en el costo total de las obras (Rojas \& Arenas, 2008).

En el periodo 2015-2016 la producción mundial de acero crudo aumentó a una tasa promedio de $0,8 \%$. Entre los países con la mayor tasa de crecimiento destacan Irán con el $11,2 \%$, India con el 7,4\% e Italia con el 6,4\%. Mientras que Japón y Estados Unidos decrecen a razón de $0,3 \%$ y $0,4 \%$ como promedio anual (Minería en línea, 2017). Los cuatro países con mayor importación de acero en el mundo son: Estados unidos con 31,5, Unión Europea con 29,5, Alemania con 22,9 y Corea del Sur con 20,4 millones de toneladas y las principales empresas productoras de acero en el mundo son: AcerolorMittal con 93,6; Nippon Steel con 47,9; Hebei Group con 42,8 y Baosteel Group con 42,7 millones de toneladas (Nahúat et al., 2014).

En Latinoamérica se describe un crecimiento ralentizado en los últimos diez años donde tan solo entre el 2004 al 2012 pasó de 64 millones de toneladas a 68 millones de toneladas siendo los mayores productores, Brasil con 35,2, México 18,2, y Argentina con 5,6. México es uno de los líderes en Latinoamérica y ubica en el décimo tercer productor a nivel global, mantiene una producción anual de 18,9 millones de toneladas (mdt), posee una capacidad instalada de producción de 28,8 mdt, exporta 5,8 mdt., e importa 12,4 mdt. (Restrepo \& Taborda, 2017; Morales et al., 2015).

Dada la importancia de la producción y comercialización del acero en el mundo, se establece que los inventarios en cada una de sus áreas juegan un papel importante en el crecimiento de la industria siderúrgica. Los inventarios son aprovisionamientos de materias primas, componentes, productos en proceso y productos terminados, que aparecen en numerosos puntos a lo largo del canal de producción y en la logística de una empresa. Los inventarios se encuentran en lugares como almacenes, patios, pisos de maniobras, equipos de transporte y en los estantes de las tiendas de menudeo. 
Los costos de mantener inventarios están asociados con la cantidad del stock almacenado. La representación del costo del inventario se encuentra entre el $30 \%$ y el $35 \%$ del valor de la empresa (Angudelo \& López, 2018). Las principales causas de mantener inventarios en cualquier empresa, es el desfase existente entre la demanda de los consumidores y la producción o suministro de dichos productos y las fluctuaciones aleatorias de la demanda y de los tiempos de reposición en la cadena de suministro. Se reconoce al inventario como un amortiguador entre dos sistemas: uno de oferta (producción o abastecimiento) y otro de demanda (clientes o distribuidores). De estas características, se entiende que el tamaño del inventario depende del comportamiento de estos sistemas (Asencio et al., 2017).

La eficacia del sistemas de control de inventarios o gestión de políticas, dependen de factores como: la medición adecuada de los tiempos de reabastecimiento, el diseño de indicadores capaces de considerar todas la variables implícitas y en especial de la implementación de métodos que permitan una estimación precisa de la tendencia y variabilidad de la demanda (Aguirre et al., 2015). Así mismo, el inventario afecta los activos que se conservan, los costos en que se incurre, y la capacidad de respuesta prevista en la cadena de suministro.

Algunos autores han establecido sus estrategias de aplicación y determinación en políticas de inventarios, tal es el caso de Salas (2017) quien establece una estrategia de integración y colaboración bajo un enfoque de mejora continua que incluye cinco pasos 1) Definición de políticas, 2) Planificación colaborativa, 3) Integración de procesos claves y críticos, 4) Medición del desempeño y 5) Elaboración de planes de acción.

Por otra parte, Chamorro (2018) determino políticas de inventario a razón de considerar máximos y mínimos en los niveles de inventarios, apoyado de la desviación estándar para representar la variabilidad de la demanda, a fin de garantizar que las existencias estén dentro de un rango establecido, donde la regla de decisión es que la posición del inventario es revisada periódicamente y la orden es puesta únicamente si el inventario está por debajo del nivel mínimo.

Otra política de inventario está definida por la consideración de riesgo, estableciendo criterios de evaluación que se tendrán en cuenta para cada ítem, asignándole un peso de importancia a través de los expertos, posteriormente utiliza una plantilla de priorización de riesgo y por ultimo realiza la estratificación de los ítems mediante clasificación ABC (Martínez, 2018).

Es posible incrementar la productividad operativa cuando se utiliza control y administración de los inventarios a través de diversas herramientas. Viveros \& Salazar (2010) han logrado un $3,6 \%$ de incremento productivo con una reducción de costos en un $5 \%$, debido al mejor uso de la capacidad y de la disminución de los inventarios a lo largo del horizonte de planificación. Para ello elaboraron un plan de trabajo para la planificación de producción, a través de un modelo de programación lineal, el cual fue implementado en MS Excel con el uso de Premium Solver Platform. En esta dirección Orejuela et al., (2014) han obtenido la reducción del 4\% del costo semanal de residuos al disminuir desperdicios de materia prima para minimizar los niveles de inventario. En esta investigación, se implementó un método matemático en lenguaje de programación AMPL de cinco fases, empleado como herramienta computacional el solucionador Gurobi del NEOS Server for Optimization.

Barrionuevo, (2010) han presentado la propuesta de mejorar el proceso de despacho que produce y comercializa acero dimensionado, mediante el análisis de problemas en las actividades logísticas, así como apoyo en los requerimientos de la demanda, para atender a los clientes y satisfacer sus necesidades al mínimo costo posible permitiendo minimizar los costos logísticos y generando el retorno del capital invertido a lo largo de toda la cadena de suministro.

Un modelo económico-matemático en el que ha trabajado Hernández (2015) ha logrado que el valor del inventario de $55 \%$ y $60 \%$ disminuyera hasta un $47,68 \%$, utilizando un sistema 
informático abastecido con " $\mathrm{n}$ " productos en períodos fijos de planeación con demanda determinista y dinámica, costos por mantener inventario y generación cada orden de compra.

Los modelos EOQ y (q, R), destacan por el importante compromiso entre los costos fijos y los costos de mantener el inventario en la venta y distribución de productos, estos modelos buscan minimizar la suma de ambos con el propósito de satisfacer la demanda sobre un horizonte de tiempo específico, por lo que las empresas realizan esfuerzos, no solo para gestionarlos debidamente, sino para que además los resultados en cuanto a eficiencia y controlabilidad del problema sean los más satisfactorios posibles.(Causado, 2015; Pérez y Torres, 2014; Ríos et al., 2008). Por ello es indispensable establecer políticas cuyos procedimientos a establecer sean fácil de entender y de aplicación, que permitan una operación constante y fluida en la empresa (Izar \& Méndez, 2013)

En el ramo acerero, se implementan modelos de inventarios que han permitido minimizar y optimizar sus costos en el manejo de materiales, permitiendo una operación controlada en la escala de la cadena de suministro. De acuerdo a lo expuesto, se presentan antecedentes teóricos de aplicación de los modelos EOQ y $(q, R)$ en empresas relacionadas con el sector de interés, a fin de conocer situaciones reales en donde se verifican aportes al tema, con el fin de facilitar el manejo y comprensión de casos más complejos.

Moina y Tapia (2016) trabajando con el modelo EOQ han logrado disminuir los costos operacionales de adquisición, manejo de inventarios y por faltantes en un $2,83 \%$ anual a través de aplicar punto de reorden con demanda incierta, calculo del tiempo de rotación del inventario, y establecimiento del ciclo de compra-venta.

En Guayaquil - Ecuador, Se presenta un caso de aplicación técnico, del modelo EOQ calculando la cantidad económica de pedido, punto de reorden, stock de seguridad y cobertura de los productos, generando un estudio de los niveles de inventario durante el periodo 2014-2015, identifica en el inventario un excedente del $20 \%$ en toneladas, con una cobertura del nivel de stock de 44,79 meses donde el objetivo de cobertura es de 4 meses. En la aplicación del modelo se logra reducir el $90 \%$ el nivel de inventario, cumpliendo las expectativas de la empresa (Santamaría, 2016).

Dada la importancia del acero para la construcción, se estima que la correcta selección de herramientas de planificación y control de inventarios pueden ser de gran utilidad para las organizaciones y contribuir a incrementar los indicadores de comercialización y elevar el servicio al cliente.

El objetivo de esta investigación es la aplicación de los modelos de inventarios EOQ y (q, R) para establecer políticas de inventario en todos los productos comercializados por la empresa, donde el algoritmo de implementación está definido por: 1. Recuperación de información sobre el comportamiento estadístico de ventas del periodo (2012-2016). 2. Identificación de unidad de medida de comercialización (Toneladas-Piezas), para su clasificación e identificación del comportamiento de su demanda. 3. Asignación de modelo de aplicación por producto de acuerdo a la identificación de su coeficiente de variabilidad. 4. Aplicación de modelos en todos los productos y obtención de las políticas de inventario. 5. Estado comparativo de los resultados de las políticas de inventario aplicadas en el primer semestre 2017 en contraste al periodo sin políticas de inventario primer semestre 2016. Se orienta a la aplicación de modelos matemáticos con enfoque de administración de inventarios, los cuales se dividen en dos grandes grupos: Modelos determinísticos y modelos estocásticos (probabilísticos), de acuerdo a la probabilidad de predecir si la demanda es constante o presenta fluctuaciones (Ramírez et al., 2009). 


\section{MATERIALES Y METODOS}

La presente investigación aplica modelos de inventario en una empresa comercializadora de aceros para la construcción a fin de establecer las cantidades óptimas requeridas a través de los modelos (Economic Order Quantity -EOQ) y el modelo de revisión continua con demanda incierta - con desviación estándar ( $q, R)$, siendo modelos matemáticos adaptables a empresas que solo basan sus decisiones de inventario en la experiencia y que requieren soportarlas para minimizar sus costos de almacenamiento.

Para este caso de estudio se consideran 3 tipos de costos que coexisten en los inventarios. 1. Costos de Flujos, que consideran el transporte de las mercancías, 2. Costos por stocks, los cuales engloban el inventario de mercancías desde su almacenamiento, deterioro, pérdidas hasta la degradación de productos almacenados, 3. Costos de procesos, enfocados a los costos por compras, lanzamiento de pedidos y por trámites (Pérez, 2017).

Atendiendo a estos tres tipos de costos se asegura la productividad y los costos claves en la gestión eficiente del almacenamiento, evitando que una mala administración de éstos y del inventario, pueden ocasionar pérdidas de tiempo, problemas de comunicación entre departamentos, reducción de productos obsoletos, errores al identificar productos y mala optimización de los recursos humanos (E Revista Énfasis Logística, 2016).

Las políticas de inventario establecidas dan respuesta a las preguntas de: cada cuando debe revisarse el inventario, cuando y cuanto ordenar, ya sean ítems de demanda independiente o dependiente previendo que la metodología aplicada estime la menor variabilidad de error. Por lo tanto, se aplican los modelos orientados a estimar la cantidad de pedido y el punto de reorden y la cantidad económica de pedido $E O Q$ y $(Q, R)$.

En la aplicación de estos modelos se estableció como primer paso identificar el Coeficiente de Variabilidad (CV), para establecer la probabilidad de demanda de la muestra y asignar el modelo acorde a su comportamiento probabilístico o determinístico.

Para toma de decisión, en la aplicación del modelo probabilístico y/o determinístico se basó en la estimación del coeficiente de variabilidad (CV). Cuando el coeficiente determinado fue menor a 0,20 se aplicó un modelo determinístico y para otros valores se consideró un modelo probabilístico (Palacios et al., 2015).

En las ecuaciones 1, 2, 3 se describe el proceso matemático para calcular el coeficiente de variabilidad:

1.- Calculo de la estimación đ de la demanda promedio por periodo:

$$
\mathrm{d}=\frac{1}{n} \sum_{i=1}^{i=n} d i
$$

2.- Calculo de la estimación de la varianza $D$ por periodo:

$$
\text { Est. } \operatorname{var} D=\frac{1}{n} \sum_{1=1}^{i=n} d i^{2}-\mathrm{d}^{2}
$$

3.- Calculo de un estimado de la variabilidad relativa de la demanda llamado coeficiente de variabilidad. A esta cantidad se le presenta como CV:

$$
C V=\frac{\text { est.var } D}{\mathrm{~d}^{2}}
$$




\section{Modelo determinístico}

Cálculo del Modelo Determinístico de Lote Económico de Pedido (EOQ).

1.- Cantidad óptima a ordenar:

$$
q * \sqrt{\frac{2 k d}{h}}
$$

2.- Costo total logístico de inventario:

$$
\mu(q) \frac{k d}{q}+c d+\frac{h q}{2}
$$

3.- Número de órdenes anuales:

$$
n=\left(\frac{d}{q^{*}}\right)
$$

4.- Duración del ciclo de pedido:

$$
T *=\frac{q^{*}}{d}
$$

Dónde:

d : Demanda determinística

$\mathrm{q}^{*}$ : Tamaño óptimo de cada pedido

$\mathrm{K}$ : Costo de ordenar

$\mathrm{h}$ : Costo de sostener inventario unitario

c : Costo de compra unitario

\section{Modelo probabilístico}

Cálculo del Modelo revisión continúa con demanda incierta y con desviación estándar (q, R) (Ríos et al., 2009).

1.- Cantidad optima a ordenar:

$$
q^{*}=\sqrt{\frac{2 k d}{h}}
$$

2.- Cálculo del punto de reorden $\mathrm{R}$ :

$$
R=d^{*} L+Z_{C S L} * S^{\prime} d
$$


3.- Costo total logístico de inventario:

$$
\mu(q) \frac{k d}{q}+c d+\frac{h q}{2}
$$

4.- Nivel de servicio por piezas:

$$
F R=1 \frac{S^{\prime} d^{*} E_{z}}{q}
$$

Dónde:

S'd: Desviación estándar de la demanda durante el tiempo de entrega ordenar

h : Costo de sostener inventario

c : Costo de compra

u : Costo de stockout (costo de faltante)

$\mathrm{E}_{z}$ : Proporción de stockout (faltante), dada Z definido.

$Z_{C S L}:$ Valor de $Z$ para la distribución normal con un nivel de servicio en el ciclo (CSL)

Para este modelo se aplica un stock de seguridad $=Z_{C S L}{ }^{*} S^{\prime d}$ (desviación estándar ajustada)

Para la aplicación de los modelos de inventario, se presenta en la Tabla 1 la cual presenta el catálogo de materiales comercializados por la empresa, y la unidad de medida de rotación dado la importancia para su consideración y aplicación en los modelos.

Tabla 1. Materiales comercializados por la empresa.

\begin{tabular}{cccl}
\hline No. & Materiales & Medida & \multicolumn{1}{c}{ Proveedor } \\
1 & Armex 20x15 & Piezas & Villacero S.A de C.V \\
2 & Armex $15 \times 15$ & Piezas & Villacero S.A de C.V \\
3 & Lamina 2,44 & Piezas & Ternium México S.A de C.V \\
4 & Lamina 3,05 & Piezas & Ternium México S.A de C.V \\
5 & Lamina 3,66 & Piezas & Ternium México S.A de C.V \\
6 & Lamina 4,27 & Piezas & Ternium México S.A de C.V \\
7 & Lamina 4,88 & Piezas & Ternium México S.A de C.V \\
8 & Lamina 5,50 & Piezas & Ternium México S.A de C.V \\
9 & Lamina 6,10 & Piezas & Ternium México S.A de C.V \\
10 & Malla 610 & Piezas & De Acero, S.A de C.V \\
11 & Malla 666 & Piezas & De Acero, S.A de C.V \\
12 & Plafón 500 & Piezas & De Acero, S.A de C.V \\
13 & Alambre & Toneladas & Ternium México S.A de C.V \\
14 & Alambrón & Toneladas & Ternium México S.A de C.V \\
15 & Clavo & Toneladas & Industrial Sobasa, S.A de C.V \\
16 & Varilla 1" VHM & Toneladas & Hylsa S.A de C.V \\
17 & Varilla 1/2 Gasa & Toneladas & Grupo Acerero S.A de C.V \\
18 & Varilla 1/2 VHP & Toneladas & Ternium México SA de C.V \\
19 & Varilla 3/8 Gasa & Toneladas & Grupo Acerero S.A de C.V \\
20 & Varilla 3/8 VHP & Toneladas & Ternium México S.A de C.V \\
\hline & Fuente. Elaboración propia de datos obtenidos de la empresa. \\
\hline
\end{tabular}


De acuerdo a los materiales se presentan los porcentajes de venta por unidad de medida comercializada. Se define aplicar la metodología en todos los materiales a fin de obtener datos cuantitativos unitarios y totales para una evaluación comparativa de mejora.

La Figura 1 muestra los porcentajes de venta de los materiales comercializados por tonelada en un periodo de análisis de 5 años, identificando que entre los materiales la varilla $3 / 8$, varilla $1 / 2$ y alambrón generan el $77 \%$ de las ventas.

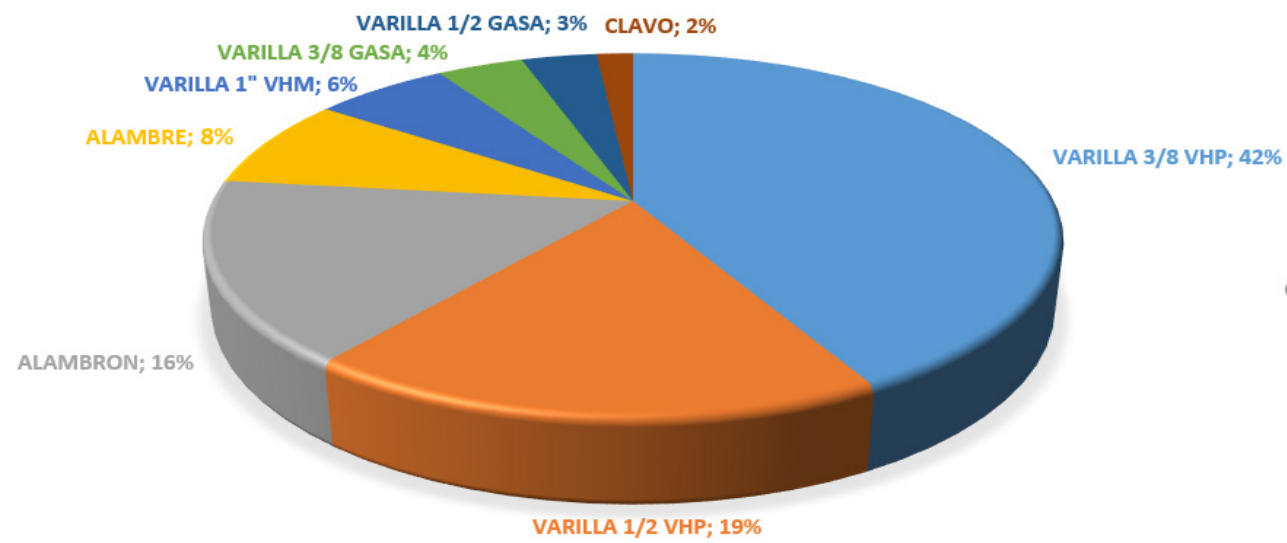

Figura 1. Distribución porcentual de las ventas de los materiales comercializados por tonelada.

Fuente: Elaboración propia con datos obtenidos de la empresa.

Con respecto a los porcentajes de ventas de los materiales comercializados en piezas representados en la Figura 2. Se identifica que el material Armex 15 X20 genera el 91\% de las ventas totales en piezas.

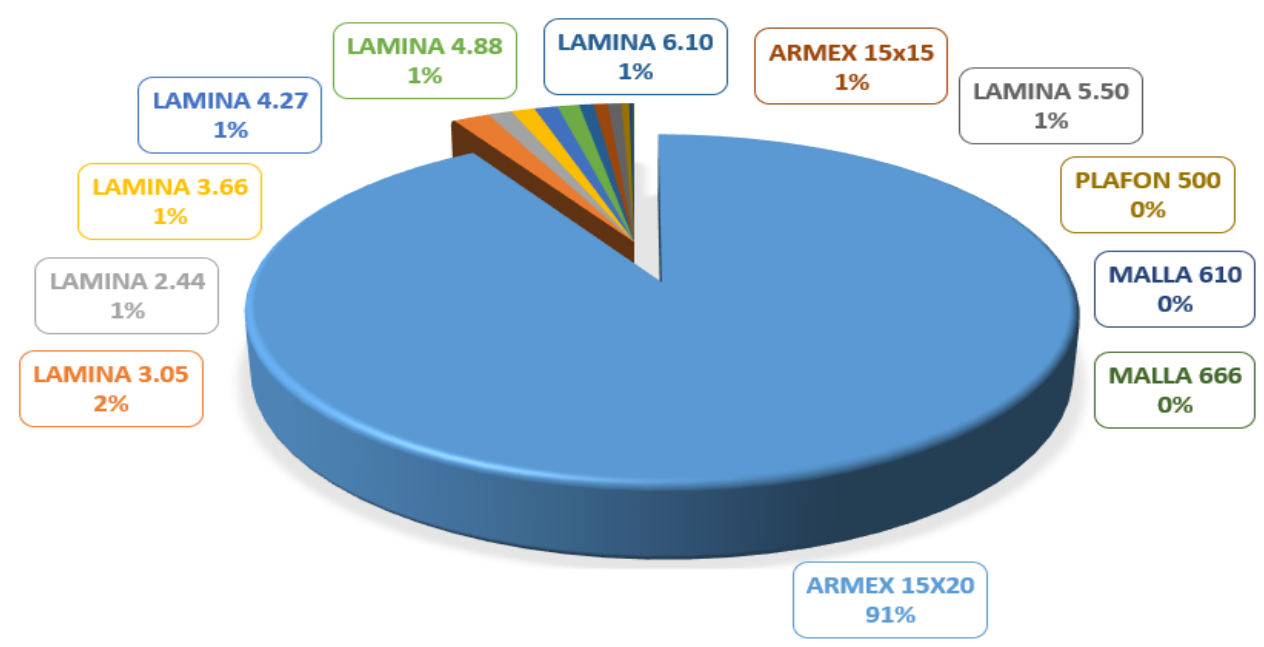

Figura 2. Distribución porcentual de ventas de los materiales comercializados por pieza.

Fuente: Elaboración propia con datos obtenidos de la empresa. 


\section{Caso de estudio}

El estudio realizado a la empresa muestra que no cuenta con la aplicación de metodologías para el control de la logística de inventarios, lo cual afecta directamente a los clientes, externando quejas en un promedio de $35 \%$ en incumplimiento de fechas y horarios de entrega de los materiales y un $27 \%$ inconformidades por entregas incompletas en sus pedidos, identificando la pérdida del 12\% de los clientes en 2016.

El retraso por incumplir con los requerimientos de los clientes, genera un $45 \%$ de re trabajos, afectando en salidas tardías de reparto, con un $60 \%$ de tiempo excedente y genera un $85 \%$ de tiempo extra en mano de obra del departamento operativo (Logística, Transporte y Almacén). Se estima un incremento del 35\% de gastos administrativos (Llamadas telefónicas, papelería, salarios administrativos, re facturaciones, generación de nuevas órdenes de pedido, servicio al cliente y combustible).

De una forma general se estima que la falta de políticas de inventario genera un gasto mayor del $45 \%$ del presupuesto mensual equivalente a US\$28 536,58, sin considerar las ventas no generadas por los clientes perdidos.

La investigación define establecer políticas de inventario que minimicen los costos logísticos en los niveles de inventario para una mejor planeación y control, que apoye en el cumplimiento de la demanda solicitada, así como en la recuperación de la credibilidad de entrega de pedidos al cliente.

Para generar las políticas de inventario se utilizan los modelos matemáticos presentados en el apartado de materiales y métodos una vez analizado el comportamiento de la demanda de los materiales.

El cálculo del coeficiente de variabilidad, elimina la dimensionalidad de las variables obtenida por la desviación estándar, como unidad de medida absoluta de la dispersión de la muestra, que expresa la variación de las unidades de los materiales demandados en el periodo de estudio. Pero no puede ser la única base para la comparación de las distribuciones de las demandas por la variabilidad. De lo mencionado si se tiene una desviación estándar de 20 unidades en cualquier material y una media de 10 , los valores varían al doble de la media. Si en otra muestra tenemos una desviación estándar de 20 con una media de 6000 , la variación respecto a la media es insignificante. En este caso se requiere una medida relativa, que nos proporcione una estimación de la magnitud de la desviación de la muestra de los materiales, respecto de la magnitud de la media. El coeficiente de variación es una medida relativa de dispersión, que expresa a la desviación estándar como un porcentaje de la media, así de esta forma se elimina la dimensionalidad de las muestras de los materiales y los agrupa a una distribución más homogénea y se define el modelo de aplicación propuestos (EOQ) ó $(Q, R)$.

La aplicación de la metodología se extiende a todos los materiales comercializados por la empresa, para interpretar las políticas de inventario obtenidas, las cuales se toman como ejemplo en la aplicación de dos materiales diferentes por la variación de los modelos, considerando la Varilla de $3 / 8$ VHP con modelo (EOQ) y Varilla de $1 / 2$ VHP para el modelo $(Q, R)$. 


\section{RESULTADOS}

La Tabla 2 presenta el modelo que aplica por material de acuerdo al coeficiente de variabilidad obtenido de la aplicación de las formulas 1, 2 y 3, descritas en la sección de metodología.

Tabla 2. Asignación del modelo para establecer la política de inventario.

\begin{tabular}{|c|c|c|c|c|}
\hline No. & Materiales & $\begin{array}{c}\text { Promedio de demanda anual } \\
\text { (Piezas) }\end{array}$ & Cv & Modelo \\
\hline 1 & Armex $15 \times 20$ & 247285 & 0,59 & Probabilístico \\
\hline 2 & Lamina 3,05 & 1206 & 0,81 & Probabilístico \\
\hline 3 & Lamina 2,44 & 641 & 1,12 & Probabilístico \\
\hline 4 & Lamina 3,66 & 1058 & 0,66 & Probabilístico \\
\hline 5 & Lamina 4,27 & 1090 & 0,96 & Probabilístico \\
\hline 6 & Lamina 4,88 & 1230 & 0,84 & Probabilístico \\
\hline 7 & Lamina 6,10 & 737 & 0,72 & Probabilístico \\
\hline 8 & Armex $15 \times 15$ & 247285 & 0,59 & Probabilístico \\
\hline 9 & Lamina 5,50 & 765 & 1,02 & Probabilístico \\
\hline 10 & Plafón 500 & 208 & 0,26 & Probabilístico \\
\hline 11 & Malla 610 & 55,85 & 1,02 & Probabilístico \\
\hline 12 & Malla 666 & 55,85 & 1,02 & Probabilístico \\
\hline No. & Materiales & $\begin{array}{l}\text { Promedio de demanda anual } \\
\text { (Toneladas) }\end{array}$ & Cv & Modelo \\
\hline 13 & Varilla 3/8 VHP & 1133 & 0,13 & Determinístico \\
\hline 14 & Varilla 1/2 VHP & 2944 & 0,31 & Probabilístico \\
\hline 15 & Alambrón & 2579 & 0,23 & Probabilístico \\
\hline 16 & Alambre & 1218 & 0,08 & Determinístico \\
\hline 17 & Varilla 1" VHM & 1313 & 0,66 & Probabilístico \\
\hline 18 & Varilla 3/8 Gaza & 1133 & 0,13 & Determinístico \\
\hline 19 & Varilla 1/2 Gaza & 1155 & 0,24 & Probabilístico \\
\hline 20 & Clavo & 245 & 0,16 & Determinístico \\
\hline
\end{tabular}

Fuente: Elaboración propia de resultados obtenidos de las formulas 1, 2 y 3.

La Tabla 3 presenta los resultados de la aplicación del modelo EOQ, a través de las formulas 4, 5, 6 y 7, para el material VARILLA 3/8 VHP, la cual establece la política de la cantidad optima a ordenar en el pedido, la cual se establece en 62,09 toneladas, de esta forma cuando el nivel de inventario llegue a 12,43 toneladas se debe realizar el siguiente pedido para asegurar que en todo momento de operación se cuente con inventario, se determina un tiempo de 19,99 días de duración del periodo de entrega, por parte del proveedor hacia la empresa en cuestión, estimando un total de pedidos de 18,26 en el año 2017. El ciclo de aplicación de los modelos se representa gráficamente en la Figura 3. 
Tabla 3. Resultados de la aplicación del modelo de lote económico.

\begin{tabular}{|c|c|c|c|c|c|c|}
\hline \multicolumn{7}{|c|}{ Modelo de Lote económico de pedido (Economic Order Quantity- EOQ) } \\
\hline No. & Material & $\begin{array}{c}\text { Cantidad } \\
\text { optima a } \\
\text { ordenar en } \\
\text { toneladas } \\
\left(q^{*}\right)\end{array}$ & $\begin{array}{c}\text { Número } \\
\text { de } \\
\text { pedidos } \\
\text { por año } \\
\text { (n) }\end{array}$ & $\begin{array}{c}\text { Tiempo } \\
\text { en días } \\
\text { entre } \\
\text { solicitud } \\
\text { de } \\
\text { pedidos } \\
\left(T^{*}\right)\end{array}$ & $\begin{array}{c}\text { Costos } \\
\text { logísticos } \\
\text { de } \\
\text { inventario } \\
\text { totales US\$ } \\
\text { (mu) }\end{array}$ & $\begin{array}{c}\text { Punto de } \\
\text { reorden en } \\
\text { toneladas (R.) }\end{array}$ \\
\hline 1 & $\begin{array}{c}\text { VARILLA } 3 / 8 \\
\text { VHP }\end{array}$ & 62,09 & 18,26 & 19,99 & 629248,01 & 12,43 \\
\hline
\end{tabular}

Fuente: Elaboración propia.

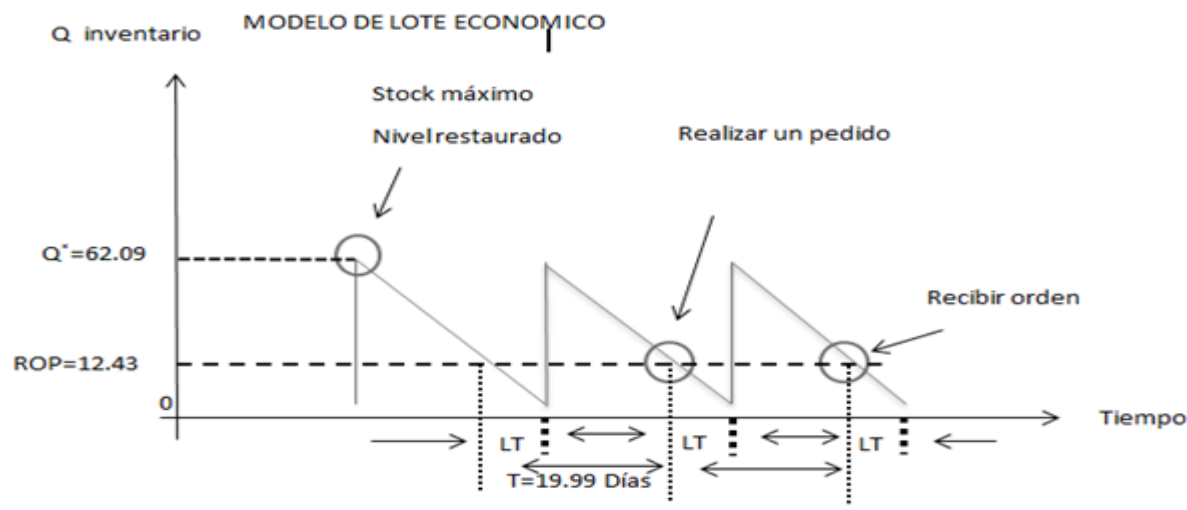

Figura 3. Representación gráfica del modelo Lote económico de pedido

(Economic Order Quantity- EOQ)

Fuente: Elaboración propia.

Para el caso de aplicación del modelo $(Q, R)$, se generan las políticas para material varilla $1 / 2$ representado en la Tabla 4 y Figura 4 . Indica que la cantidad óptima a ordenar será de 4,88 toneladas, identificando que cuando el nivel de inventario llegue a 25,56 toneladas será el punto de reorden, generando costos logísticos totales de inventario por US\$136 159,34, garantizando un nivel de servicio del $99 \%$, esta política nos permite asegurar entregas completas de los pedidos del cliente.

Tabla 4. Resultados de la aplicación del modelo de revisión continúa con demanda incierta y con desviación estándar.

Modelos de revisión continua con demanda incierta - con desviación estándar (q,R)

\begin{tabular}{ccccccc} 
No. & Material & $\begin{array}{c}\text { Cantidad } \\
\text { optima a } \\
\text { ordenar en } \\
\text { toneladas (q*) }\end{array}$ & $\begin{array}{c}\text { Punto de } \\
\text { reorden en } \\
\text { toneladas } \\
\text { (R.) }\end{array}$ & $\begin{array}{c}\text { Costos } \\
\text { logísticos de } \\
\text { inventario } \\
\text { totales US\$ } \\
\text { (mu) }\end{array}$ & $\begin{array}{c}\text { Nivel de } \\
\text { servicio } \\
\text { (\%) }\end{array}$ & $\begin{array}{c}\text { Stock de } \\
\text { seguridad } \\
\text { (Piezas) }\end{array}$ \\
\hline 1 & $\begin{array}{c}\text { Varilla 1/2 } \\
\text { VHP }\end{array}$ & 4,88 & 25,56 & 136159,34 & 99 & 0,04 \\
\hline
\end{tabular}

Fuente: Elaboración propia. 


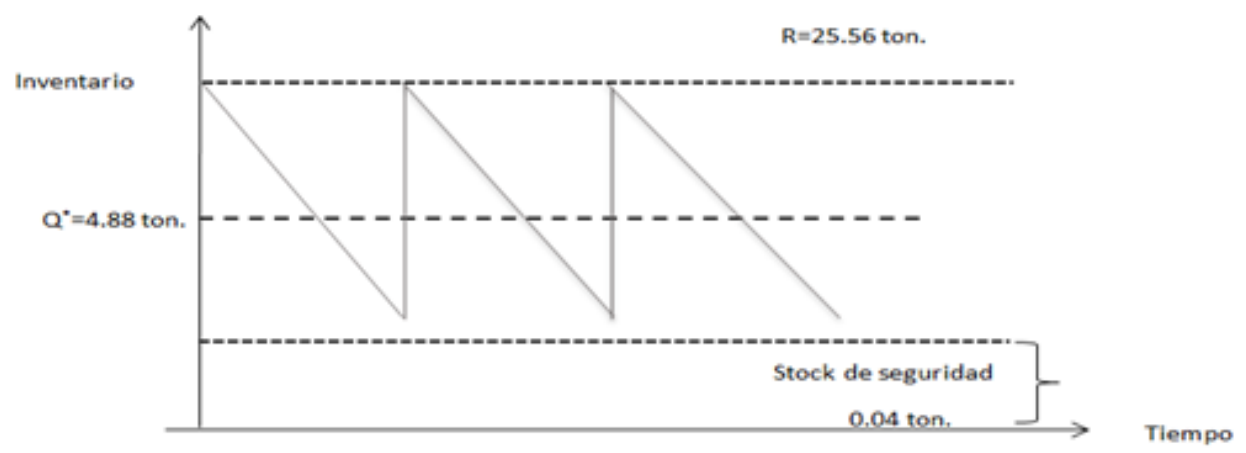

Figura 4. Representación gráfica del modelo de revisión continúa con demanda incierta y con desviación estándar $(\mathrm{Q}, \mathrm{r})$ del material Varilla $1 / 2$ VHP. Fuente: Elaboración propia.

En las Tablas 5, 6 y 7 se presentan las políticas de inventario obtenidas para cada uno de los materiales comercializados por la empresa, los cuales se determinaron de acuerdo a la forma de comercialización por tonelada o pieza.

Tabla 5. Políticas de inventario obtenidas de la aplicación del modelo de lote económico para materiales comercializados en toneladas.

\begin{tabular}{|c|c|c|c|c|c|c|}
\hline No. & Material & $\begin{array}{l}\text { Cantidad } \\
\text { optima a } \\
\text { ordenar en } \\
\text { toneladas }\left(q^{*}\right)\end{array}$ & $\begin{array}{l}\text { Número de } \\
\text { pedidos } \\
\text { por año }(n)\end{array}$ & $\begin{array}{c}\text { Tiempo en } \\
\text { días entre } \\
\text { solicitud } \\
\text { de pedidos } \\
\left(T^{*}\right)\end{array}$ & $\begin{array}{l}\text { Costos } \\
\text { logísticos } \\
\text { de } \\
\text { inventario } \\
\text { totales US\$ } \\
\text { (mu) }\end{array}$ & $\begin{array}{c}\text { Punto de } \\
\text { reorden en } \\
\text { toneladas } \\
\text { (R.) }\end{array}$ \\
\hline 1 & Varilla $3 / 8$ VHP & 62,09 & 18,26 & 19,99 & 629248,01 & 12,43 \\
\hline 2 & Alambre & 64,35 & 18,93 & 19,28 & 984814,41 & 13,35 \\
\hline 3 & Varilla 3/8 Gaza & 62,09 & 18,26 & 19,99 & 530968,03 & 12,43 \\
\hline 4 & Clavo & 28,86 & 8,49 & 43,00 & 187352,92 & 2,68 \\
\hline
\end{tabular}

Fuente: Elaboración propia.

Tabla 6. Políticas de inventario obtenidas del modelo de revisión continúa con demanda incierta y con desviación estándar para materiales comercializados en toneladas.

Modelos de revisión continua con demanda incierta - con desviación estándar (q,R)

\begin{tabular}{|c|c|c|c|c|c|c|}
\hline No. & Material & $\begin{array}{l}\text { Cantidad } \\
\text { optima a } \\
\text { ordenar en } \\
\text { toneladas } \\
\left(q^{*}\right)\end{array}$ & $\begin{array}{c}\text { Punto de } \\
\text { reorden en } \\
\text { toneladas } \\
\text { (R.) }\end{array}$ & $\begin{array}{l}\text { Costos } \\
\text { logísticos de } \\
\text { inventario } \\
\text { totales US\$ } \\
\text { (mu) }\end{array}$ & $\begin{array}{c}\text { Nivel de } \\
\text { servicio } \\
\text { (\%) }\end{array}$ & $\begin{array}{c}\text { Stock de } \\
\text { seguridad } \\
\text { (Piezas) }\end{array}$ \\
\hline 1 & Varilla 1/2 VHP & 4,88 & 25,56 & 136159,34 & 99 & 0,04 \\
\hline 2 & Alambrón & 4,61 & 21,5 & 117038,38 & 99 & 0,10 \\
\hline 3 & Varilla 1" VHM & 3,24 & 10,96 & 61309,91 & 99 & 0,05 \\
\hline 4 & Varilla 1/2 Gaza & 3,1 & 9,64 & 52078,18 & 99 & 0,14 \\
\hline
\end{tabular}

Fuente: Elaboración propia. 
Tabla 7. Políticas de Inventario obtenidas de la aplicación del modelo de revisión continúa con demanda incierta y con desviación estándar para materiales comercializados en piezas.

\begin{tabular}{|c|c|c|c|c|c|c|}
\hline \multicolumn{7}{|c|}{ Modelos de revisión continua con demanda incierta - con desviación estándar (q,R) } \\
\hline No. & Material & $\begin{array}{l}\text { Cantidad } \\
\text { optima a } \\
\text { ordenar en } \\
\text { piezas }\left(q^{*}\right)\end{array}$ & $\begin{array}{c}\text { Punto de } \\
\text { reorden } \\
\text { en piezas } \\
\text { (R.) }\end{array}$ & $\begin{array}{c}\text { Costos } \\
\text { logísticos de } \\
\text { inventario } \\
\text { totales US\$ } \\
\text { (mu) }\end{array}$ & $\begin{array}{c}\text { Nivel de } \\
\text { servicio (\%) }\end{array}$ & $\begin{array}{l}\text { Stock de } \\
\text { seguridad } \\
\text { (Piezas) }\end{array}$ \\
\hline 1 & Armex $15 \times 20$ & 16 & 6 & 99,22 & 96 & 0,27 \\
\hline 2 & Lamina 3,05 & 27 & 37 & 734,82 & 86 & 0,04 \\
\hline 3 & Lamina 2,44 & 22 & 25 & 319,57 & 87 & 0,05 \\
\hline 4 & Lamina 3,66 & 24 & 28 & 70,407 & 88 & 0,06 \\
\hline 5 & Lamina 4,27 & 22 & 38 & 926,31 & 81 & 0,04 \\
\hline 6 & Lamina 4,88 & 22 & 39 & 1212,97 & 81 & 0,04 \\
\hline 7 & Lamina 6,10 & 15 & 21 & 891,16 & 86 & 0,07 \\
\hline 8 & Armex 15x15 & 18 & 6 & 75,84 & 97 & 0,27 \\
\hline 9 & Lamina 5,50 & 16 & 28 & 839,42 & 81 & 0,05 \\
\hline 10 & Plafón 500 & 10 & 5 & 172,81 & 95 & 0,32 \\
\hline 11 & Malla 610 & 2 & 2 & 318,10 & 88 & 0,68 \\
\hline 12 & Malla 666 & 1 & 2 & 634,94 & 84 & 0,68 \\
\hline
\end{tabular}

La Figura 5, muestra un estado comparativo sobre los trabajos e indicadores afectados por la falta de políticas de inventario en 2016 con respecto a lo obtenido en el establecimiento de políticas de inventario en 2017 , presentando una disminución del $65 \%$ en el tiempo extra de mano de obra, reduciendo las salidas tardías de un $60 \%$ a un $15 \%$, mejorando el flujo y carga de los materiales, agilizando la salida para el reparto a los clientes, con lo que se reducen un $28 \%$ las entregas incumplidas y un $22 \%$ las entregas incompletas, disminuyendo así los re trabajos en un $4 \%$ y presentando perdidas del cliente de un $12 \%$ a un $2 \%$.

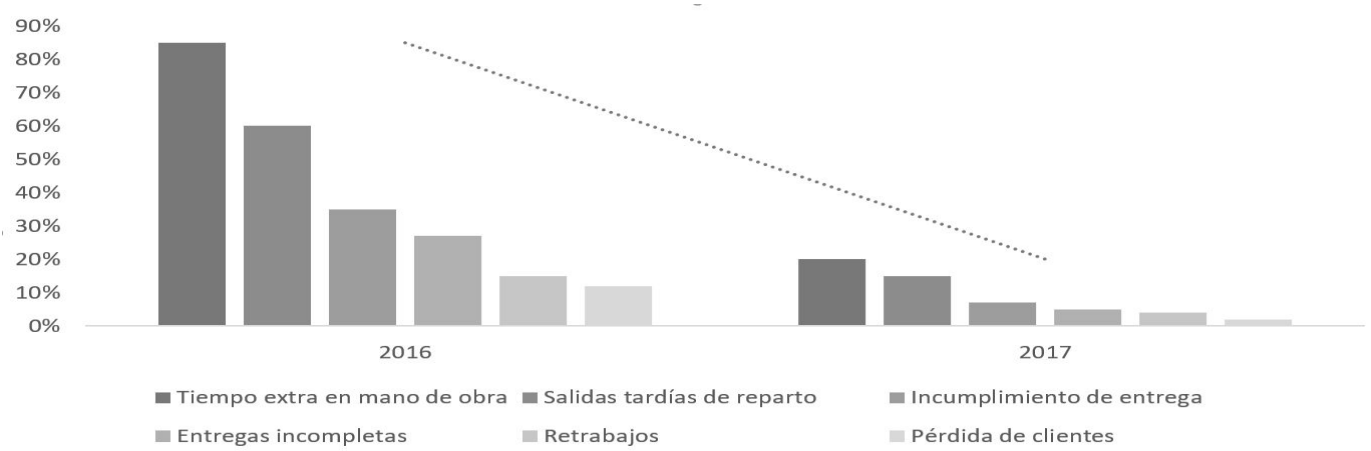

Figura 5. Comparación del caso de estudio del primer semestre 2016 Vs 2017.

La Figura 6, presenta de una forma porcentual la disminución del excedente del prepuesto gastado en el primer semestre del 2016 vs 2017 , el cual del $45 \%$ se reduce a un $15 \%$, obteniendo una reducción del 30\%. 


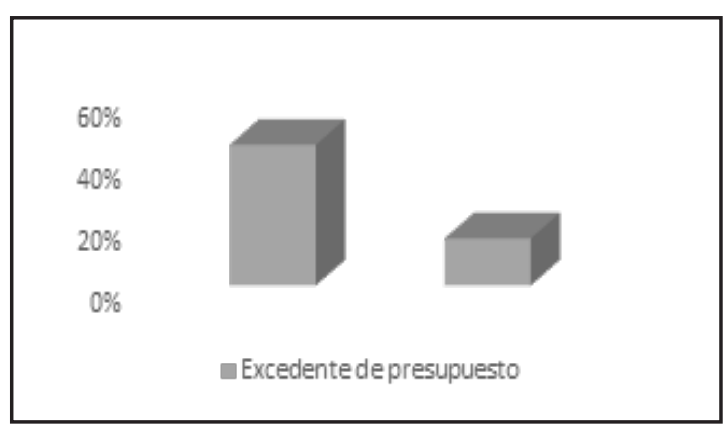

Figura 6. Reducción del excedente de presupuesto del primer semestre de 2017 versus 2016.

Se reduce el 30\% con respecto al 2016 el cual equivale a un ahorro económico para el primer semestre del año 2017 de US\$145 536,56, más de lo que se destina al presupuesto de operación de la empresa para un periodo de 60 días.

\section{DISCUCIÓN}

De acuerdo a los resultados obtenidos, al aplicar el modelo ABC a los materiales de acero resulta evidente que cuatro, son los productos más rentables para la empresa los cuales son: Varilla $3 / 8$, Varilla $1 / 2$, Alambrón, y Armex $15 X 20$. Por lo tanto, a estos materiales se les debe de aplicar un alto nivel de seguimiento, al momento de establecer las políticas de inventario correspondientes, para determinar cuándo se deben pedir más productos a los proveedores con el fin de no tener desabastecimientos en el almacén, pero tampoco tenerlos en exceso, evitando así pérdidas, a fin de generar una mayor utilidad a la comercializadora de aceros.

Establecer políticas de inventario para los materiales es sin duda un gran paso para la administración de la empresa y su reducción de costos, sin embargo la relevancia en esta investigación es la aplicación de la técnica de Coeficiente de Variabilidad para eliminar la dimensionalidad de las muestras en su demanda y agruparlos en una distribución homogénea, para determinar de acuerdo a su comportamiento el modelo de inventario que ha de aplicarse en el establecimiento de políticas de inventarío, identificando de entre los 20 materiales comercializados aplicar en 16 con el modelo probabilístico equivalente al Modelo de Lote Económico EOQ y a 4 el Determinístico el cual corresponde el (q,R) con demanda incierta.

De esta forma en la aplicación del modelo EOQ se generaron políticas de inventario: "para la cantidad óptima a ordenar, el número de pedidos por año, tiempo en días entre solicitud de pedidos, costos logísticos de inventario totales y el punto de reorden". En la aplicación del modelo $(q, R)$ se generaron políticas de inventario: "para la cantidad optima a ordenar, punto de reorden, costos logísticos de inventarios totales, nivel de servicio y stock de seguridad.

Así mismo se identifica que en los múltiples casos de empresas presentados como marco referencial en la aplicación de políticas de inventario en el sector acerero, enfocan solo esfuerzos en aplicar políticas en demandas de comportamiento homogéneo, con un enfoque exclusivamente al desabasto de los inventarios, a diferencia del caso de estudio presentado en este artículo se generan políticas de inventario dentro de un análisis primeramente ajustando la metodología de acuerdo a un análisis de comportamiento individual por producto y buscando el mínimo error en la definición de políticas para los inventarios a fin de disminuir los costos logísticos totales de inventario a través de los tiempos de pedido, la identificación 
del número total de pedidos al año, identificación del punto de reorden, estableciendo un stock de seguridad y garantizando un \% de seguridad en la atención al cliente a través de entregas completas de los materiales en el tiempo requerido por el cliente.

Se reconoce la carencia de estudios metodológicos en la gestión de políticas de inventario en el sector siderúrgico para la construcción, por lo tanto se invita a los lectores a profundizar en el tema y aportar conocimientos en la gestión de políticas inventarios que ayuden a minimizar el riesgo de faltantes o sobrantes en los materiales, a fin de cumplir con el $100 \%$ de los pedidos al mínimo de costo con alto nivel de servicio.

\section{CONCLUSIONES}

El establecer políticas de inventario a través de los modelos de lote económico de pedido (Economic Order Quantity -EOQ) y revisión continúa con demanda incierta (q, R), orientó a los administradores de almacén a elaborar una planeación logística de los inventarios, identificando con seguridad los volúmenes de pedido, garantizando las demandas de los clientes sin generar excedente, acertando el nivel de servicio en un 90,4\% recuperando credibilidad y la recuperación del $10 \%$ de clientes.

Los modelos aplicados atendieron la necesidad de establecer políticas para el tamaño de pedido con el fin de proyectar los pedidos requeridos en un periodo de 12 meses, calendarizando la solicitud de cada pedido e identificando el punto de reorden, estableciendo presupuestos para sus costos logísticos de inventario, identificando que la planeación establecida de acuerdo a las políticas de inventario minimizó el número de viajes de entrega a los clientes por re surtimientos por falta de productos, estas políticas permitieron generar un rol de entrega de los materiales, estableciendo que del $100 \%$ de ahorro obtenido de la aplicación de dichas políticas, el 65\% corresponde a ahorros en el consumo de combustible y mano de obra por re trabajos y tiempo extra.

Con los resultados obtenidos se confirma que la aplicación de métodos cuantitativos para generar y establecer políticas de inventario genera resultados positivos en beneficio de las organizaciones y que estas no están limitadas a un sector comercial, por lo que la industria del acero para la construcción requiere de estas metodologías en apoyo a minimizar sus costos logísticos de inventario para lograr el aumento de su rentabilidad.

Como trabajo futuro se pretende buscar y aplicar otras metodologías que ayuden a mejorar los resultados ya obtenidos, como la simulación y las tecnologías de información aplicadas a la logística de inventarios.

\section{REFERENCIAS}

AGUIRRE LASPRILLA, S., ARDILA RUEDA, W., FIGUEROA, L. y ROMERO RODRÍGUEZ, D. H. (2015). Parametrización y evaluación de Política de Inventario (s,Q) en Hospitales: Un caso de estudio en la ciudad de barranquilla. Prospectiva [en línea]. 13(1), 99-105 [consulta: 18 de marzo de 2018]. ISSN 1692-8261. Disponible en: http://www.redalyc.org/ pdf/4962/496250641010.pdf 
ANGUDELO SERNA, D. A. y LÓPEZ RIVERA, Y. M. (2018). Dinámica de sistemas en la gestión de inventarios. Revista de ingenierías USBMED [en línea]. 9(1), 75-85, [consulta: 28 de junio de 2018]. ISSN 2027-5846. Disponible en: https://dialnet.unirioja.es/servlet/ articulo?codigo $=6283786$.

ASENCIO CRISTÓBAL, L., GONZÁLEZ ASCENCIO, E. y LOZANO ROBLES, M. (2017). EI inventario como determínate en la rentabilidad de las distribuidoras farmacéuticas. Revista de Ciencias de la Administración y Economía [en línea]. 13(7), 123-142, [consulta: 23 agosto de 2018]. ISSN 1390-8618. Disponible en:_http://scielo.senescyt.gob.ec/scielo.php?script=sci_ arttext\&pid=S1390-86182017000100231\&lng=es\&nrm=iso

BARRIONUEVO CASTILLO. J. K. (2010). Propuesta de mejora del proceso de despacho en una empresa que produce y comercializa acero dimensionado [en línea]. GIORDANO, C. E (tutor). Trabajo fin de carrera. Universidad Peruana de Ciencias Aplicadas, 2010 [consulta: 24 de junio de 2018]. Disponible en: http://dx.doi.org/10.6084/m9.figshare.1594842

CAUSADO RODRÍGUEZ, E. (2015). Modelo de inventarios para control económico de pedidos en empresa comercializadora de alimentos. UMC: Revista Ingenierías [en línea]. 14(27),163-177, [consulta: 10 de diciembre de 2017]. ISSN 1692-3324. Disponible en: https:// revistas.udem.edu.co/index.php/ingenierias/article/view/1692/1748

CHAMORRO COREA, J. L., DIAZ CAMEJO, J. E., FUENTES ESPINOZA, O. D. Y LOVO GUTIERREZ, H.Y. (2018). Política de inventarios máximos y mínimos en cadenas de suministro multinivel. caso de estudio: una empresa de distribución farmacéutica. Nexo Revista Científica [en línea]. 31(2), 144-156, [consulta: 02 de abril de 2019]. ISSN-e 19959516. Disponible en: http://dx.doi.org/10.5377/nexo.v31i2.6837

HERNÁNDEZ GONZÁLEZ, C. J. 2015. Metodología para la coordinación del inventario de materias primas y materiales en la industria de astilleros. Anuario Facultad de Ciencias Económicas y Empresariales [en línea]. 6, 60-80, [consulta: 16 febrero de 2018]. ISSN 22183639. Disponible en: https://revistas.uo.edu.cu/index.php/aeco/article/viewFile/256/250

IZAR, J. M. y MENDEZ, H. (2013). Estudio comparativo de la aplicación de 6 modelos de inventarios para decidir la cantidad y el punto de reorden de un artículo. UASLP: Revista Ciencia y Tecnología [en línea]. 13, 217-232, [consulta: 10 de abril de 2018]. ISSN 18500870. Disponible en: https://dialnet.unirioja.es/servlet/articulo?codigo $=4843863$

E Revista Énfasis Logística. (2016). Descuidar almacén ocasiona pérdidas a empresas. [en línea], [consulta: 11 de enero de 2018]. Disponible en:http://www.logisticamx.enfasis.com/ notas/76798-descuidar-almacen-ocasiona-perdidas-empresas

MARTÍNEZ ESCOBAR, N. y OSORIO GÓMEZ, J.C. (2018). Gestión de inventarios de repuestos considerando el riesgo. Revista Espacio [en línea]. 39(44), 29-29, [consulta: 03 de abril de 2019]. ISSN 0798 1015. Disponible en: http://www.revistaespacios.com/a18v39n44/ a18v39n44p29.pdf

MINERÍA EN LíNEA. (2017). Producción Mundial de Acero Crece, con una Participación Mundial de China de 49,6\%. [en línea]. [consulta: 17 de marzo de 2018]. Disponible en: https://mineriaenlinea.com/2017/07/produccion-mundial-acero-crece-una-participacionchina-49-6/

MOINA CANDO, N. G. y TAPIA MOGROVEJO, J. T. (2016). Análisis y propuesta de un modelo de gestión de inventarios para el sector comercial ferretero industrial (Ertalo, acero, rodamiento) de acuerdo a la denominación INEC G4719.00. En la ciudad de Cuenca. Caso 
de aplicación: Empresa el acero. Periodo 2015-2016 [en línea]. Campoverde Jiménez, P. P. (tutor). Trabajo fin carrera Ingeniería. Universidad de Cuenca, [consultado: 05 de octubre de 2017]. Disponible en: http://dspace.ucuenca.edu.ec/bitstream/123456789/25540/1/ Trabajo\%20de\%20Titulaci\%C3\%B3n.pdf

MORALES FAJARDO, M. E., GALEANA ESTRADA, A. y MEJIA LOPEZ, M. (2015). La industria siderúrgica en México. Universidad Autónoma del Estado de México. Revista de Análisis de Coyuntura Económica. Economía Actual [en línea]. 9(2), 3-7 [consulta: 30 de agosto de 2018]. ISSN 2007-0993. Disponible en: http://web.uaemex.mx/feconomia/ Publicaciones/e902/EA9-2_03_Esther_Morales.pdf

NÁHUAT ARREGUÍN, J. J., BLANCO JIMÉNEZ, M. y BUENROSTRO NÚÑEZ, D. (2014). Propuesta teórica de los factores estratégicos que fomentan la internacionalización de la industria del acero en el noroeste de México. Revista Innovaciones de Negocios [en línea]. 11,117-142, [Consulta: 10 de octubre de 2017]. ISSN 2007-1191. Disponible en: http://eprints. uanl.mx/12599/1/11.21\%20Art6\%20pp\%20117\%20-\%20142.pdf

OREJUELA CABRERA, J. P., PEÑA CALDERÓN, D. A. y GIL GONZÁlEZ, C. A. (2014). Reducción de desperdicios de materia prima en una empresa del sector metalmecánico mediante la implementación y modelación del cutting stock problema. Revista Producción + Limpia [en línea]. 9(1), 73-90, [consulta: 14 de enero de 2018]._ISSN 1909-0455. Disponible en: http://www.scielo.org.co/pdf/pml/v9n1/v9n1a06.pdf

PALACIOS GARCÍA, L., FLORES MIRANDA, L., ÁLVAREZ VARGAS, R., CONTRERAS JUÁREZ, A., SÁNCHEZ PARTIDA, D. y MARTINEZ FLORES, J.L. (2015). Gestión de Inventario a Través de Push \& Pull e Inventario Manejado por el Proveedor - En Productos Risa, S.A. de C.V. Revista DYNA Management [en línea]. 3(1), 1-15, [consulta 24 de junio de 2018]. Disponible en: http://dx.doi.org/10.6036/mn7565.

PÉREZ MEDELLÍN, D. (2017). ¿Un Inventario equivale a gasto o inversión? Bogotá: Revista de Logística [en línea]. [consulta: 11 de diciembre de 2017]. Disponible en: https:// revistadelogistica.com/actualidad/un-inventario-equivale-a-gasto-o-inversion/

PÉREZ MANTILLA, F. A. y TORRES, F. (2014). Modelos de inventarios con productos perecederos: revisión de literatura. Revista Ingeniería [en línea]. 19 (2), 9-40, [consulta 02 de marzo de 2019]. ISSNe 2344-8393. Disponible en: http://www.scielo.org.co/pdf/inge/v19n2/ v19n2a01.pdf

RAMÍREZ NAYIBE, L., SOTO, D., PÉREZ, M. y GAMEZ, W. (2009). Aplicación de un modelo de inventarios multiproducto para las PYMES en Bogotá. Facultad de ingeniería de la universidad libre. Revista Ingenio Libre [en línea]. 9(8), 74-81, [consulta: 23 de enero de 2018]. ISSN 1692-0767. Disponible en: http://www.unilibre.edu.co/revistaingeniolibre/revista9/articulos/Aplicacion-de-un-modelo-de-inventarios-multiproducto-para-las-PYES-en-Bogota.pdf

RESTREPO CARVAJAL, C. A. y TABORDA OSORIO, J. F. (2017). El mercado colombiano del acero estructural en el contexto de la globalización. Revista Ciencias Estratégicas [en línea]. 25(38), 339-359, [consulta: 30 de agosto de 2018]. ISSN: 2390-0024. Disponible en: http://www.redalyc.org/articulo.oa?id=151354939005

RÍOS, F., MARTÍNEZ, A., PALOMO, T., CÁCERES, S. y DÍAZ, M. (2009). Inventarios Probabilísticos con Demanda Independiente de Revisión Continua, Modelos con Nuevos Pedidos. CIENCIA ergo-sum, Revista Científica Multidisciplinaria de Prospectiva [en línea]. 15(3), 251 258, [consulta 25 de abril de 2018]. ISSNe: 1405-0269. Disponible en: http://www.redalyc.org/ pdf/104/10415303.pdf 
ROJAS LOPEZ, M. D. y ARENAS GIRALDO, J. J. (2008). Comparación técnico-financiera del acero estructural y el hormigón armado. Revista DYNA [en línea]. 75 (155), 47-56, [consulta: 28 de agosto de 2018]. ISSN: 0012-7353. Disponible en: https://revistas.unal.edu.co/index. php/dyna/article/view/1739/11577

SALAS NAVARRO, K., MAIGUEL MEJIA, H. y ACEVEDO CHEDID. (2017). Metodología de Gestión de Inventarios para determinar los niveles de integración y colaboración en una cadena de suministro. Chile: Revista chilena de ingeniería [en línea]. 25 (2), 326-337, [consulta: 03 de abril de 2019]. ISSNe: 0718-3305. Disponible en: https://scielo.conicyt.cl/ scielo.php?script=sci_arttext\&pid=S071833052017000200326

SANTAMARÍA HEREDIA, G. B. (2016). Desarrollo de un modelo de manejo de inventarios para la materia prima de la empresa acero [en línea]. E. Desintonio, (dir. Informe de proyecto integrador). Escuela superior politécnica del litoral. Facultad de Ingeniería en Mecánica y Ciencias de la Producción, [consultado: 17 octubre de 2018]. Disponible en: https://www. dspace.espol.edu.ec/retrieve/93763/D-CD88284.pdf

VASQUEZ GRANADOS, A. y ABARCA GUERRERO, L. (2018). Trazabilidad de la varilla de acero para construcción \#3 en ArcelorMittal Costa Rica. Revista Tecnología en Marcha [en línea]. 31(1), 131-143, [consulta: 12 de mayo de 2018]. Disponible en: https://doi. org/10.18845/tm.v31i1.3503

VIVEROS, R. y SALAZAR, E. (2010). Modelo de planificación de producción para un sistema multiproducto con múltiples líneas de producción. Revista Ingeniera de Sistemas [en línea]. 24, 89-102 [consulta: 16 de Marzo de 2018]. Disponible en: http://www.dii.uchile. cl/ ris/RISXXIV/Viveros89.pdf 